\title{
Ceruloplasmin activity and iron chelation treatment of patients with Parkinson's disease
}

\author{
Guillaume Grolez 2,3, Caroline Moreau 2,3, Bernard Sablonnière", Guillaume Garçon ${ }^{5}$, Jean-Christophe Devedjian 1,3, \\ Sayah Meguig ${ }^{1}$, Patrick Gelé ${ }^{6}$, Christine Delmaire ${ }^{3,7}$, Regis Bordet ${ }^{1,3}$, Luc Defebvre ${ }^{2,3}$, loav Z Cabantchik ${ }^{8}$ \\ and David Devos ${ }^{1,2,3,5^{*}}$
}

\begin{abstract}
Background: Growing body of evidence suggests that Parkinson's disease (PD) is associated with oxidative damage via iron accumulation in the substantia nigra (SN). Low ceruloplasmin (CP)-ferroxidase activity has been identified in the SN and the cerebrospinal fluid (CSF) of patients with PD. The iron chelator, deferiprone, reduces the abnormally high levels of iron in the SN. In order to determine CP's involvement in iron accumulation in SN and PD progression, we aim to compare the ability of iron chelation treatment to reducing both SN iron levels and motor handicap in PD patients according to the level of ceruloplasmin activity.

Methods: We used a moderate chelation protocol with deferiprone (DFP) based on a, 6-month delayed-start paradigm, randomized placebo controlled clinical trial in 40 PD patients. CP-ferroxidase activity was determined in blood and CSF together with the D544E gene polymorphism (rs701753). Iron levels were determined by R2* MRI sequence and the motor handicap by the UPDRS motor score.
\end{abstract}

Results: After 6 to 12 months of DFP treatment, greater reductions in SN iron levels and UPDRS motor scores were obtained in patients with higher serum and CSF levels of CP-ferroxidase activity. After 6 months of DFP treatment, the AT genotype group displayed greater reduction of iron level in the SN with greater CSF and serum levels of CP activity than the AA genotype group.

Conclusion: Although most of the DFP-treated patients displayed clinical and radiological improvements, those with the lower CP activity appeared to respond better to iron chelation. Larger RCTs are now needed to establish whether pharmacological modulation of CP activity could be an innovative neuroprotective strategy in PD.

Trial registration: FAIR-PARK study (ClinicalTrials.gov reference: NCT00943748; French national reference number: 2008-006842-25). This study was approved by the French Drug Agency (ANSM) and the local institutional review board ("Comité de Protection des Personnes of Lille").

Keywords: Parkinson's disease, Iron chelation, L-dopa, Motor response, Deferiprone

\section{Background}

The oxidative damage associated with extrahepatic siderosis is generally attributed to excessive generation of reactive oxygen species following a rise in labile cell iron $[1,2]$. The latter generally attains toxic levels as a result of imbalances between iron ingress, use, storage and

\footnotetext{
* Correspondence: david.devos@chru-lille.fr

'Department of Medical Pharmacology, Lille Nord de France University, Lille, France

${ }^{2}$ Department of Movement Disorders and Neurology, Lille Nord de France University, Lille, France

Full list of author information is available at the end of the article
}

egress. Cell iron egress and thereby cell iron accumulation appear to be modulated by ceruloplasmin (CP), a copper ferroxidase that facilitates the incorporation of plasma iron (II) into circulating apotransferrin. Ceruloplasmin's key role is illustrated by an extreme example: that of aceruloplasminemia, which affects both systemic and brain iron metabolism. Low CP-ferroxidase activity has also been identified in the substantia nigra (SN) and the cerebrospinal fluid (CSF) of patients with Parkinson's disease (PD) [3-5], and particularly in individuals bearing the AT genotype of the rs707753 CP gene variant (corresponding to a D544E protein change) [2]. A

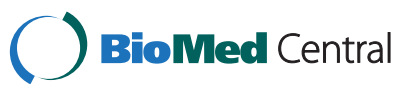

(C) 2015 Grolez et al.; licensee BioMed Central. This is an Open Access article distributed under the terms of the Creative Commons Attribution License (http://creativecommons.org/licenses/by/4.0), which permits unrestricted use, distribution, and reproduction in any medium, provided the original work is properly credited. The Creative Commons Public Domain Dedication waiver (http://creativecommons.org/publicdomain/zero/1.0/) applies to the data made available in this article, unless otherwise stated. 
screening of ceruloplasmin gene sequences variations allowed to identify five new missense variations and an already known variation (rs707753), which was not previously identified in PD. Of these six missenses variations, D544E polymorphism was the most frequent and the only one to be significantly associated with PD and iron overload measured in SN by transcranial ultrasound [6]. Although a growing body of evidence suggests that $\mathrm{PD}$ is associated with oxidative damage via iron accumulation in the SN [7], CP's putative involvement in iron accumulation and disease progression remains subject to debate. One way of addressing this issue involves comparing the ability of moderate iron chelation treatment to reducing both SN iron levels and United Parkinson's Disease Rating Scale (UPDRS) scores in PD patients according to the level of ceruloplasmin activity. The underlying rationale is that PD patients with lower $\mathrm{CP}$ activity might retain more cell iron and thus respond more favorably to iron chelation therapy.

\section{Methods}

To test this hypothesis, we used a moderate chelation protocol with deferiprone (DFP, administered at $30 \mathrm{mg} /$ $\mathrm{kg}$ /day in two daily doses) that had previously served as the basis for a translational study (i.e. including a preclinical study with cells and mice models and the first double-blind, placebo-controlled, randomized clinical trial of an iron chelator in PD) $[8,9]$.

\section{The FAIR-PARK study}

(ClinicalTrials.gov reference: NCT00943748; French national reference number: 2008 - $006842-25$ ) was based on a 6-month delayed-start paradigm in $40 \mathrm{PD}$ patients on stable dopamine regimens. Treatment with DFP was initiated at 0 or 6 months and stopped at 18 months. In the initial phase, 40 early-stage PD patients were randomly assigned to receive either oral liquid DFP (15 mg/ $\mathrm{kg}$ in the morning and evening) for 18 months (the ES group) or placebo for 6 months and then DFP for 12 months (the DS group) (Table 1).

\section{Study approval}

All clinical investigations were performed in accordance with the tenets of the Declaration of Helsinki. All patients provided their written, informed consent to participation. The aims and procedures of the main study and a compassionate 12-month extension was approved by the French Drug Agency (ANSM) and the local institutional review board ("Comité de Protection des Personnes of Lille").

\section{Motor handicap}

(i.e. disease progression) was assessed using the UPDRS motor score.

\section{Magnetic resonance imaging}

Was performed with multiple-echo/spin-echo sequences in a 3-Tesla MRI system, in order to estimate the R2\% proton relaxation rates $(=1 / \mathrm{T} 2 \%)$ and thus levels of

Table 1 Neurological characteristics of the patients on study entry (overall and by genotype, AT vs. AA)

\begin{tabular}{|c|c|c|c|c|}
\hline & \multirow{2}{*}{$\begin{array}{l}\text { Delayed-start group } \\
\text { Total }\end{array}$} & \multicolumn{3}{|c|}{ Early-start group } \\
\hline & & Total & AT genotype & AA genotype \\
\hline Number of patients & 19 & 21 & 5 & 14 \\
\hline Age at study entry (years) & $60[54-64]$ & $61[54-66]$ & $62[61-62]$ & $61[53-66]$ \\
\hline Gender (F/M) & $6 / 13$ & $9 / 12$ & $3 / 2$ & $6 / 10$ \\
\hline Time since diagnosis (years) & $2[1-3]$ & $2[1-3]$ & $2[1-3]$ & $2[1-3]$ \\
\hline Hoehn and Yahr stage (range, 1-5) & $2[1.5-2]$ & $2[1.5-2]$ & $2[1.5-2]$ & $2[1.4-2]$ \\
\hline Daily L-dopa dose equivalent (mg) & $300[162-510]$ & $300[150-532]$ & $300[150-532]$ & $300[150-532]$ \\
\hline Patients on L-dopa (n) & 9 & 10 & 3 & 7 \\
\hline Patients on dopaminergic agonists ( $\mathrm{n}$, mean daily L-dopa equivalent in mg) & $18(243)$ & $18(220)$ & $4(225)$ & $13(250)$ \\
\hline Patients on L-dopa and agonists (n) & 7 & 7 & 2 & 5 \\
\hline Patients on rasagiline $(\mathrm{n})$ & 3 & 3 & 1 & 2 \\
\hline Mini Mental Scale Examination score & $29[28-30]$ & $28[26-30]$ & $28[27-29]$ & $28[26-30]$ \\
\hline Mattis Dementia Rating Scale score & $139[138-141]$ & 139 [134-143] & $139[138-140]$ & 139 [133-143] \\
\hline Epworth Sleepiness Scale score & $9[7-13]$ & $9[5-13]$ & $8[6-10]$ & $9[5-13]$ \\
\hline Montgomery Asberg Depression Rating Scale & $3[2-4]$ & $4[2-5]$ & $3[2-5]$ & $4[2-5]$ \\
\hline Cognition and behavior: MDS-UPDRS part I score & $6[5-8]$ & $6[4.7-9]$ & $5[4-7]$ & $6[4.7-9]$ \\
\hline
\end{tabular}

There were no significant intergroup differences. The dopaminergic drug regimens did not change over the course of the study. Quantitative variables are quoted as the median [interquartile range]. MDS means Movement Disorders Society. 
agglomerated iron in a surface analysis of the SN (performed with the RelaxMaps tools in the Philips Research Imaging Development Environment ${ }^{\circ}$.

\section{The sample size}

Was based on R2* measurements in the $\mathrm{SN}$. We estimated that with an alpha risk of $5 \%$ and a power of $80 \%$, 24 subjects per treatment group would be needed to detect a change in SN R2* after 6 months of DFP treatment (relative to placebo). When the correlation coefficient between consecutive measurements (at 6-month intervals) was set to 0.4 and the data were adjusted for baseline R2* levels, the required sample size fell to 20 .

\section{Clinical biochemistry assays}

Included ferritin, $\mathrm{CP}$ and $\mathrm{CP}$-ferroxidase activity in blood and CSF. Levels of CP-ferroxidase activity in CSF and sera were determined according to the method published by Schosinsky et al. (1974) [10], as modified by Martinez-Subiela et al. (2007) [11] and Siotto et al.
(2014) [12]. Briefly, the procedure is based on the CPmediated oxidation of o-dianisidine, a chromogenic substrate forming a yellow-brown reaction product that absorbs at $500 \mathrm{~nm}$. The $\mathrm{CP}$ activity is proportional to the rate of formation of reaction product. The working reagent solution was prepared immediately before use by mixing 1 volume of substrate solution (o-dianisidine dihydrochloride: $7.8 \mathrm{mM}$, Triton X-100: 1\% v/v) with 4 volumes of sodium acetate buffer solution $(100 \mathrm{mM}, \mathrm{pH}$ adjusted to 4.6). All reagents were from Sigma-Aldrich. Samples of CSF or serum $(15 \mu \mathrm{L})$ were added to $300 \mu \mathrm{L}$ of working reagent solution and the increase in absorbance at $500 \mathrm{~nm}$ over $100 \mathrm{~s}$ (first reading point: $200 \mathrm{~s}$; second reading point: $300 \mathrm{~s}$ ) at $37^{\circ} \mathrm{C}$ was read [11]. The following parameters were used to provide the results in international units per liter (IU/L), parameters we used were as follows: molar absorption coefficient of o-dianisidine oxidation product at $37^{\circ} \mathrm{C}(\varepsilon 500 \mathrm{~nm})=$ $4.83 \times 10[3] \mathrm{L} / \mathrm{mol} / \mathrm{cm}$, optical path length $=1 \mathrm{~cm}$, dilution factor $=21$, and change in time $=100 \mathrm{~s}$. Reference levels



Figure 1 Flowchart of the patients who participated to the clinical trial and to the ceruloplasmin analysis. 
of CP activity were defined using in-house standard CSF and serum samples, which were included as controls in all assays.

\section{Genetic analysis}

Genomic DNA was extracted from venous blood samples using standard procedures. The rs701753 CP gene variant (corresponding to a D544E protein change) was genotyped using a polymerase chain reaction (PCR)restriction length polymorphism assay. The total reaction volume of $25 \mu \mathrm{l}$ contained $50 \mathrm{ng}$ genomic DNA in AmpliTaq PCR buffer with one unit of ampli Taq polymerase (Applied Biosystems, CA, USA), $1.50 \mathrm{mM}$ $\mathrm{MgCl}_{2}, 250 \mu \mathrm{M}$ dNTPs, and 15 pmol of forward primer (5'-CCCCAGTTGGACTTACCTGT-3') and reverse primer (5'-GATCCTGTGTGTCTAGCTAAGATG-3'). The cycling conditions were as follows: $5 \mathrm{~min}$ at $96^{\circ} \mathrm{C}, 35$ cycles of $30 \mathrm{sec}$ at $94^{\circ} \mathrm{C}, 1 \mathrm{~min}$ at $55^{\circ} \mathrm{C}$ and $1 \mathrm{~min}$ at $72^{\circ} \mathrm{C}$, and then $5 \mathrm{~min}$ at $72^{\circ} \mathrm{C}$. Amplification products were resolved in $5 \%$ acrylamide gels. BamH1 endonuclease was used to characterize the $\mathrm{A}$ and $\mathrm{T}$ alleles.

\section{Statistical analysis}

In view of the sample size and the skewed, all quantitative variables were expressed as the median (interquartile range).

We used a covariance analysis (adjusted for baseline differences) to estimate outcomes after 6, 12 and 18 months of treatment. For non-normally distributed data, the robustness of the results was checked after log transformation. The threshold for statistical significance was set to $p<0.05$. We used Wilcoxon's signed-rank test to compare a pair of related measurements (e.g. repeated measurements on a single sample). All statistical analyses were performed with SAS software (version 9.3, SAS Institute Inc., Cary, NC).


B

D

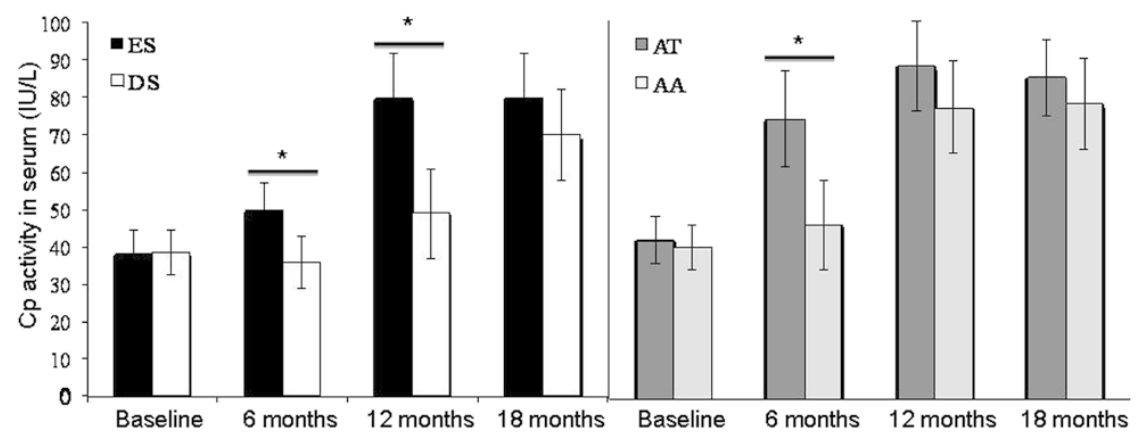

Figure 2 Ceruloplasmin activity and iron chelation treatment. (A) Effect of the iron chelator DFP on CSF levels of CP-ferroxidase activity. The patients in the ES group ( $n=11$ with two lumbar punctures: at baseline and at 6 months) displayed significantly higher CSF levels of CP activity than the patients in the DS group $\left(F_{(1,16)}=13 ; p=0.002\right.$ (B) Effect of the iron chelator DFP on serum levels of CP-ferroxidase activity. The patients in the ES group showed significantly higher serum levels of $C P$ activity than the patients in the DS group at 6 months $\left(F_{(1,35)}=26 ; p=0.0001\right)$ and 12 months $\left(F_{(1,34)}=5.2 ; p=0.028\right)$ but not at 18 months. (C) Effect of the iron chelator DFP on CSF levels of CP-ferroxidase activity as a function of D544E genotype. The DFP-treated patients with an AT genotype $(n=5)$ displayed significantly higher CSF levels of CP activity than the DFPtreated patients with an AA genotype $(n=6)\left(F_{(1,8)}=7 ; p=0.02\right)$. (D) Effect of the iron chelator DFP on serum levels of CP-ferroxidase activity as a function of D544E genotype. The DFP-treated patients with an AT genotype (AT; $n=5$ ) displayed significantly higher levels of $C P$ activity than DFP-treated patients with an AA genotype (AA; $n=15)$ at 6 months $\left(F_{(1,17)}=7 ; p=0.02\right)$ but not at 12 or 18 months. (E) Correlation between CSF levels of CP-ferroxidase activity and the R2* MRI value in the SN. The change in CSF levels of CP activity between the baseline and the visit at 12 months were significantly correlated with the change in the SN's R2* value $(r=0.784 ; p=0.001)$. White circles represent the DFP-treated patients with an AA genotype. Grey circles represent the DFP-treated patients with an AA genotype with an AT genotype; the latter displayed higher levels of $\mathrm{CP}$ activity and a greater reduction in $\mathrm{R} 2^{*}$ in the $\mathrm{SN}$. 


\section{Results}

Deferiprone reduces the abnormally high levels of iron in the SN (with little effect on systemic iron levels defining a conservative mode of iron chelation [8]) and the UPDRS motor scores (i.e. indicators of disease progression) [9]. We compared results obtained in patients having started DFP treatment $(30 \mathrm{mg} / \mathrm{kg} /$ day) early (the ES group; Figure 1) with those with a delayed start (the DS group; Figure 1). We observed that after 6 to 12 months of treatment, relatively greater reductions in $\mathrm{SN}$ iron levels and UPDRS motor scores were obtained in patients with higher serum and CSF levels of CPferroxidase activity (Figure 1, Figure 2 A\&B). The increase in serum $\mathrm{CP}$-ferroxidase activity was associated with significant higher serum $\mathrm{CP}$ levels after 12 months of DFP treatment in the ES group (from $0.28 \mathrm{~g} / \mathrm{L}$ [0.26-0.32] to $0.30 \mathrm{~g} / \mathrm{L}[0.26-0.35]$ as compared with the DS group (from 0.27 [0.24-0.29] to 0.27 [0.24-0.30]; $\mathrm{p}=0.04$ ).

We screened for the D544E gene polymorphism (rs701753: AT and AA genotypes) in the ES group; after 6 months DFP treatment, the AT group displayed greater CSF and serum levels of $\mathrm{CP}$ activity than the AA group (Figure $2 \mathrm{C} \& \mathrm{D}$ ). On the basis of median [interquartile range] R2* values in MRI (Figure $2 \mathrm{E}$ ), iron levels in the $\mathrm{SN}$ fell greater in the AT group (from 4.5 [4.2-4.9] at baseline to 3.9 [3.8-4.1] after 6 months $\left(\mathrm{F}_{(1,17)}=7.9\right.$; $\mathrm{p}=0.01)$ and 3.8 [3.6-4] after 12 months $\left(\mathrm{F}_{(1,16)}=10.1\right.$; $\mathrm{p}=0.06)$ ) than in the AA group (from 4.6 [4.4-4.8] at baseline to 4.4 [4.2-4.4] after 6 months and 4.0 [3.84.3] after 12 months).

Although the AT group displayed a greater mean reduction in the motor UPDRS score than the AA group (decreases of 4 and 3 points, respectively), the difference was not statistically significant.

\section{Discussion}

Our results suggest that iron chelation efficacy appeared to be related with the level of CP activity. PD patients with lower $\mathrm{CP}$ activity might retain more cell iron and thus respond more favorably to iron chelation therapy. Thus, CP may have a pivotal role in iron accumulation, which exacerbates the PD progression. Although most of the DFP-treated patients with PD displayed clinical and radiological improvements, those with the D544E CP polymorphism appeared to respond better to iron chelation. The greater mean reduction in the motor UPDRS score in the AT group as compared with the AA group did not reach the significant level. This might have been related to the small sample size and the many factors affecting the clinical response (notably DFP's symptomatic effect through inhibition of catechol-O-methyl transferase) [9].

Larger RCTs are now needed to establish whether (i) the D544E CP polymorphism is a bona fide biomarker for responsiveness to iron chelation therapy in selected PD patients and (ii) pharmacological modulation of CP activity, by itself, could be an innovative neuroprotective strategy in PD.

\section{Conclusion}

Lower ceruloplasmin activity in PD may be associated with iron overload in SN. Ceruloplasmin ferroxidase activity deficiency in PD could be partly improved by iron chelation by deferiprone in both groups (AA and AT) but mostly in the AT group, in which D544E polymorphism induced a lower ferroxidase activity. The measure of the R2* MRI sequence in SN was improved in both groups but the reduction was higher in the D544E (AT).

\section{Competing interests}

The authors have no financial or non-financial disclosures to make or potential conflicts of interest to report in relation to this investigator-led study.

\section{Authors' contributions}

1) The research project: $A$ : conception; B: organization; $C$ : execution 2) The manuscript: A: the first draft, B. review and critical comment G. Grolez: 1b, c and 2a; C. Moreau: 1a, b, c and 2b; B. Sablonnière 1b, c and 2b; G. Garçon 1b, c and 2b; J-C. Devedjian: 1a and 2b; S. Meguig: 1c and 2b; C. Delmaire: $1 \mathrm{~b}$ and 2b; K. Dujardin: $1 \mathrm{~b}$ and 2b; R. Bordet: $1 \mathrm{a}$ and 2b; L. Defebvre: $1 \mathrm{~b}$ and 2b; Z.I. Cabantchik 1a and 2b; D. Devos 1a, b, c and 2a, b GG participated to the organization and execution of the study and to the first draft manuscript. CM participated to the conception, the organization and the execution of the study and helped to the final review and critical comment. BS participated to the organization and the execution of the study and helped to the final review and critical comment. GG participated to the organization and execution of the study and helped to the final review and critical comment. JCD participated to the organization and helped to the final review and critical comment. SM participated to the execution of the study and helped to the final review and critical comment. CD participated to the organization of the study and helped to the final review and critical comment. KD participated to the organization of the study and helped to the final review and critical comment. RB participated to the conception of the study and helped to the final review and critical comment. LD participated to the organization of the study and helped to the final review and critical comment. ZIC participated to the conception of the study and helped to the final review and critical comment. DD participated to the conception, the organization and the execution of the study, to the first draft manuscript and helped to the final review and critical comment. All authors read and approved the final manuscript.

\section{Acknowledgments}

We thank the French Ministry of Health for funding (PHRC grants 2008 006842 - 25), ApoPharma for providing DFP and placebo formulations, the French Parkinson's Disease Association and Fédération de la Recherche Clinique du CHU de Lille for promoting the study, Dominique Deplanque and the Centre d'Investigation Clinique de Lille for data acquisition, Francine Niset, Carine Piatek, Delphine Massart and Bertrand Accart for their assistance, David Fraser (Biotech Communication, Damery, France) for editorial assistance; and AFIRNE (Paris) and the DellaPergola Chair in Life Sciences of HUJI for funding ZIC. Lastly, we thank all the study patients and their physicians for their participation.

\section{Study funding}

This academic, investigator-led study was funded by the French Ministry of Health (PHRC grant 2008 - 006842 - 25) and the French Parkinson's Disease Association. ApoPharma provided DFP formulations, placebo formulations and advices on drug use. The principal investigator (DD) had full access to all the study data and had final responsibility for submitting the study report for publication. 
Full financial disclosures of all authors for the past 5 years

Guillaume Grolez, Guillaume Garçon, Sayah Meguig, Jean-Christophe Devedjian, Christine Demaire and Bernard Sablonnière have no disclosures to report. Caroline Moreau has served on the Scientific Advisory Board for Aguettant. Kathy Dujardin has served on the Scientific Advisory Board for Novartis and received a grant from the MJ Fox Foundation for Parkinson's research. Régis Bordet receives funding from the French Ministry of Research. He has received various honoraria from pharmaceutical companies for consultancy and lectures at symposia.

Luc Defebvre has served on the Scientific Advisory Board for Novartis and Aguettant, and has received various honoraria from pharmaceutical companies (Abbott and Boehringer) for consultancy and lectures on Parkinson's disease at symposia.

Z. loav Cabantchik was funded by the A\&M Della Pergola Chair in Life Sciences, has served on the Scientific Advisory Board of Novartis ESB, has received research grants from NIH, EEC (5 and 6 framework), Israel Science Foundation, Novartis and ApoPharma in the past (and also speaker's fees from the last two) and is presently a consultant for Aferrix, Ltd and Hinoman, Ltd. David Devos served on the Scientific Advisory Board for Novartis and Aguettant. He has received research funding from the French Ministry of Health and the ARSLA charity, together with consultancy fees and speaker's fees from pharmaceutical companies but not from ApoPharma.

\section{Author details}

'Department of Medical Pharmacology, Lille Nord de France University, Lille, France. ${ }^{2}$ Department of Movement Disorders and Neurology, Lille Nord de France University, Lille, France. ${ }^{3}$ INSERM U1171, Lille Faculty of Medicine, Lille Nord de France University, Lille, France. ${ }^{4}$ Department of Molecular Biology and Pathology Centre, Unit 837, Team 1, INSERM Lille Faculty of Medicine, Lille University Hospital, Lille Nord de France University, Lille, France. ${ }^{5}$ Department of Toxicology, Public Health and Environment, EA 4483, Faculty of Pharmaceutic and Biological Sciences, Lille Nord de France University, Lille, France. ${ }^{6}$ Biological Resources Centre, Lille University Hospital, Lille, France. ${ }^{7}$ Department of Neuroradiology, Lille University Hospital, Lille, France. ${ }^{8}$ Della Pergola Chair, Alexander Silberman Institute of Life Sciences, Hebrew University, Jerusalem, Israel.

Received: 19 November 2014 Accepted: 24 April 2015

Published online: 06 May 2015

\section{References}

1. Breuer W, Shvartsman M, Cabantchik Zl. Intracellular labile iron. Int J Biochem Cell Biol. 2008:40:350-4.

2. Hochstrasser H, Tomiuk J, Walter U, Behnke S, Spiegel J, Krüger R, et al. Functional relevance of ceruloplasmin mutations in Parkinson's disease. FASEB J. 2005;19:1851-3.

3. Olivieri S, Conti A, lannaccone S, Cannistraci CV, Campanella A, Barbariga M, et al. Ceruloplasmin oxidation, a feature of Parkinson's disease CSF, inhibits ferroxidase activity and promotes cellular iron retention. J Neurosci. 2011;31:18568-77.

4. Ayton S, Lei P, Duce JA, Wong BX, Sedjahtera A, Adlard PA, et al. Ceruloplasmin dysfunction and therapeutic potential for Parkinson disease. Ann Neurol. 2013;73:554-9.

5. Boll MC, Sotelo J, Otero E, Alcaraz-Zubeldia M, Rios C. Reduced ferroxidase activity in the cerebrospinal fluid from patients with Parkinson's disease. Neurosci Lett. 1999;265:155-8.

6. Hochstrasser H, Bauer P, Walter U, Behnke S, Spiegel J, Csoti I, et al. Ceruloplasmin gene variations and substantia nigra hyperechogenicity in Parkinson disease. Neurology. 2004:63(10):1912-7.

7. Friedman A, Galazka-Friedman J. The history of the research of iron in Parkinsonian substantia nigra. J Neural Transm. 2012;119:1507-10.

8. Cabantchik ZI, Munnich A, Youdim MB, Devos D. Regional siderosis: a new challenge for iron chelation therapy. Front Pharmacol. 2013;4:167.

9. Devos D, Moreau C, Devedjian JC, Kluza J, Petrault M, Laloux C, et al. Targeting chelatable iron as a therapeutic modality in Parkinson's disease. Antioxid Redox Signal. 2014;21:195-210.

10. Schosinsky KH, Lehmann HP, Beeler MF. Measurement of ceruloplasmin from its oxidase activity in serum by use of o-dianisidine dihydrochloride. Clin Chem. 1974;20:1556-63.
11. Martınez-Subiela S, Tecles F, Ceron JJ. Comparison of two automated spectrophotometric methods for ceruloplasmin measurement in pigs. Res Vet Sci. 2007:83:12-9.

12. Siotto $M$, Pasqualetti $P$, Marano $M$, Squitti R. Automation of o-dianisidine assay for ceruloplasmin activity analyses: usefulness of investigation in Wilson's disease and in hepatic encephalopathy. J Neural Transm. 2014;121:1281-6

\section{Submit your next manuscript to BioMed Central and take full advantage of:}

- Convenient online submission

- Thorough peer review

- No space constraints or color figure charges

- Immediate publication on acceptance

- Inclusion in PubMed, CAS, Scopus and Google Scholar

- Research which is freely available for redistribution

Submit your manuscript at www.biomedcentral.com/submit 